\title{
Variations du bilan de masse dans les glaciers et prédicteurs climatiques : application aux glaciers du Groupe Ortles-Cevedale
}

\author{
Variations of the mass balance in glaciers and climate forecasting : \\ application to Ortles-Cevedale glaciers
}

\author{
par Rossi G.C.,
}

Comitato Glaciologico Italiano, Noale (VE)

Belloni S.,

Université de Milan, Département de Sciences de la Terre

Diolaiuti G., Smiraglia C.,

Université de Milan, Département de Sciences de la Terre,

Comitato Scientifico - Club Alpino Italiano

This article tries to reconstitute the net mass balance of the three sample glaciers of Ortles-Cevedale (Caresèr, Sforzellina and Fontana Bianca), italian Alps, beginning with the possible relations between measured mass balances and some climate parameters, in particular the global incident radiation. The models are then applied to temporal measurement series.

\section{I 口 INTRODUCTION}

Le travail suivant représente une tentative de reconstruction du bilan de masse net relatif aux trois glaciers témoins du Groupe Ortles-Cevedale (le Glacier du Caresèr, le Glacier de la Sforzellina et le Glacier de Fontana Bianca, figure 1), en commençant par les possibles relations des bilans de masse mesurés grâce à quelques paramètres climatiques.

\section{II — SCHÉMA DU MODÈLE}

En adoptant un schéma simplifié du bilan énergétique, valable en première approximation pour les glaciers tempérés (qui présentent donc une valeur d'ablation $\mathrm{a}_{\mathrm{a}}$ non nulle) [1], l'énergie nécessaire pour produire l'ablation dérive de trois termes fondamentaux : radiation solaire nette $(R)$, transfert turbulent de la chaleur latente (V) et transfert turbulent de la chaleur sensible $(\mathrm{H})$. Ces trois termes donnent lieu à :

$$
R+H+V=a_{a}
$$

En réunissant tous les termes radiatifs et en rendant explicite la relation avec le temps comme somme des événements, $\Sigma_{T}(R+H+V)=\Sigma_{T} Q$, on obtient une relation explicite par rapport au terme de l'ablation:

$$
\Sigma_{T} a_{a}=T / L \Sigma_{T} Q
$$

où $T$ représente la durée (en jours) de la période d'ablation, $L$ représente la chaleur latente de fusion de la glace $(334 \mathrm{~kJ} / \mathrm{kg}), \Sigma_{T} Q$ représente le flux d'énergie nette exprimé en $\mathrm{kJ} / \mathrm{m}^{2}$.

Si $T_{a}$ est la température de l'air, $T_{s}$ la température à la surface du glacier et $a_{c}$ le terme d'accumulation dans l'équation du bilan de masse net et, par la suite, si on met en relation les

\begin{tabular}{|l|l|c|c|c|c|c|}
\hline Glacier & Bassin & N. & Latitud. & Surface $\mathbf{k m}^{\mathbf{2}}$ & Pente & Exposition \\
\hline Sforzellina & Po-Adda-Gavia & $\mathbf{5 1 6}$ & 46.33 & 0.39 & 26 & NW \\
\hline Careser & Adige-Noce & $\mathbf{7 0 1}$ & 46.45 & 4.68 & 11 & SE \\
\hline Fontana B.ca & Adige-Rio Valsura & $\mathbf{7 1 3}$ & 46.48 & 0.75 & 26 & E \\
\hline
\end{tabular}

Tableau 1 -Caractéristiques géométriques des glaciers de l'Ortles-Cevedale, objets de mesure du bilan 


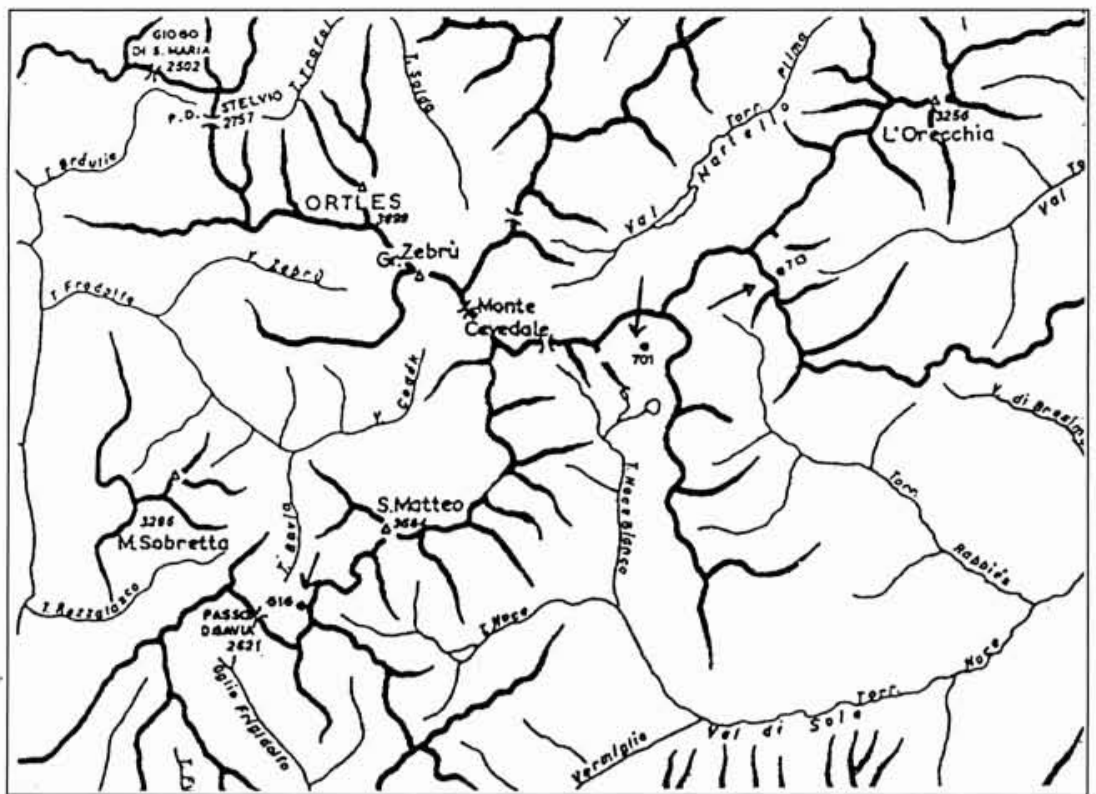

1. Le Glacier du Caresèr, $\mathrm{n}^{\circ} \mathbf{7 0 1}$; le Glacier de la Sforzellina, $n^{\circ} 516$ et le Glacier de Fontana Bianca, $\mathbf{n}^{\circ} \mathbf{7 1 3}$.

flux énergétiques de $H$ et $V$ avec $\left(T_{a}-T_{s}\right)$, pour les glaciers tempérés sous les conditions d'ablation (c'est-à-dire $T_{a}>T_{s}$ et $T_{s} \approx 0^{\circ} \mathrm{C}$ ), on obtient l'expression linéaire suivante :

$$
\Sigma_{T} b=-\Sigma_{T}\left(R-a_{c}\right)=\gamma \Sigma_{T}\left(T_{a}-T_{s}\right)
$$

où le terme $\Sigma_{T}\left(R-a_{c}\right)$ désigne la quantité d'énergie radiante accumulée du glacier pendant la période entière d'ablation pour fondre la neige d'accumulation $a_{c}$ et, éventuellement, la glace. Le terme $\Sigma_{T}\left(T_{a}-T_{s}\right)$ est donné par la somme des degré*jours $>0{ }^{\circ} \mathrm{C}$, pendant la période d'ablation de la longueur temporelle $T$ et le terme $\gamma$ représente le coefficient d'échange énergétique. Ce coefficient prend des valeurs entre 0,5 et $2,7 \mathrm{MJ} / \mathrm{m}^{2} / \mathrm{jour} /{ }^{\circ} \mathrm{C}$, avec une raisonnable valeur moyenne, pour les glaciers des Alpes, de $1,7 \mathrm{MJ} / \mathrm{m}^{2} /$ jour $/{ }^{\circ} \mathrm{C}$ (correspondant à peu près à $5 \mathrm{~kg} / \mathrm{m}^{2}$ d'équivalent en eau de glace fondue pour chaque degré*jours $>0^{\circ} \mathrm{C}$ ) (Kuhn, 1989). Pour calculer les termes radiatifs, nous avons utilisé un modèle théorique de la radiation incidente, basé sur les valeurs d'observation de l'état du ciel.

La radiation solaire incidente sur les surfaces terrestres horizontales, $K_{n}$, dérive du flux de radiation astronomique, $K_{0}$, qui traverse l'atmosphère. La valeur de $K_{0}$ est calculée à partir de la relation :

$$
K_{0}=S \sin B
$$

où $S$ représente la constante solaire, dont la valeur est $4871 \mathrm{~kJ} / \mathrm{h} / \mathrm{m}^{2}$, et l'angle $B$ désigne l'ascension droite du soleil, calculée à partir des coordonnées géographiques du lieu et de la position du soleil à partir de la date, grâce aux algorithmes de trigonométrie sphérique, pour lesquels nous renvoyons à $[2,3]$.

La radiation incidente sur un plan horizontal (directe et diffuse) dépend de la transparence de l'atmosphère et de la position du soleil, dont les effets sont représentés grâce au paramètre de transmissivité nette $T_{k}$ [4], donné par la formule suivante :
$T_{k}=(0,6+0,2 \sin B)\left(1-0,4 \sigma_{C H}\right)\left(1-0,7 \sigma_{C M}\right)\left(1-0,4 \sigma_{C L}\right)$

où $\sigma_{C H}, \sigma_{C M}$ et $\sigma_{C L}$ représentent la partie du ciel couverte par les nuages, des nuages respectivement d'altitude haute, moyenne et basse. Quand le soleil est au zénith et le ciel couvert, $T_{k}=0,80$; quand le ciel est complètement couvert à tous les niveaux d'altitude, $T_{k}=0,086$.

Les moyennes mensuelles calculées de la radiation incidente sur une surface horizontale, ont été vérifiées par les mesures d'heures d'ensoleillement relatives au Glacier du Caresèr, pendant la période 1975-1996, et par les mesures d'intensité du flux de la radiation, pendant la période 19901994. La moyenne d'ensoleillement pendant cette période est de 1755 heures/an, où celle relative aux quatre mois juinoctobre couvre à peu près $50 \%$ du total, alors que la radiation moyenne pour la même période est à peu près de $13000 \mathrm{~kJ} / \mathrm{m}^{2}$. Le calibrage a donné des résultats très positifs ; en effet, la dispersion obtenue, entre les données expérimentales et les données calculées, est interpolée par une parabole dont l'équation est la suivante :

$Y=-1259+1,3708949 X-2,4507$ E-5 $X^{2} \quad\left(R^{2}=0,971\right)$

Cette équation représente les valeurs mesurées en fonction des valeurs calculées. Les valeurs moyennes de $K_{n}$, (correctes) ont été utilisées pour calculer la radiation incidente sur une surface inclinée par rapport à l'horizon d'un angle égal à celui de la pente moyenne de la surface du glacier examiné, et d'exposition relative et représentées à l'aide de l'expression suivante:

$$
K_{t}=R K_{n}
$$

où le facteur $\mathrm{R}$ représente le rapport entre la radiation incidente sur une surface inclinée et celle incidente sur une surface horizontale, toutes deux exposées au Sud. L'expression de $R$ est la suivante :

$R=\left(1-K_{d} / K_{n}\right) R_{b}+K_{d} / K_{n}(1+\cos s) / 2+<(1-\cos \mathrm{s}) / 2$ 
2. Modèle à 3 variables du Glacier de Caresèr - Graphique de dispersion des valeurs simulées/mesurées.

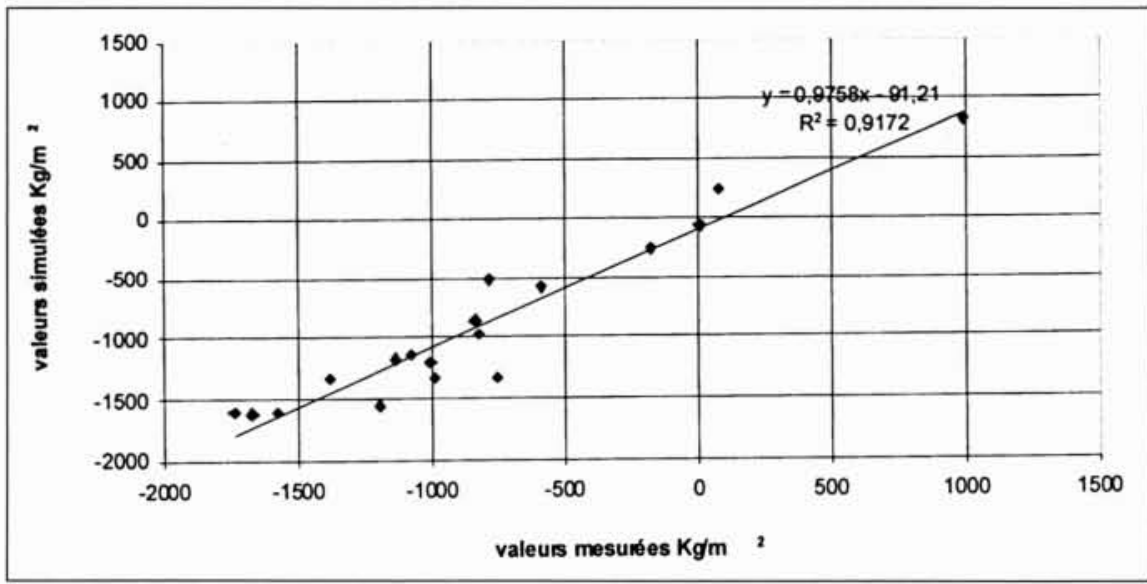

3. Modèle à 2 variables de la Vedretta Caresèr - Graphique de comparaison des séries des valeurs simulées/mesurées.

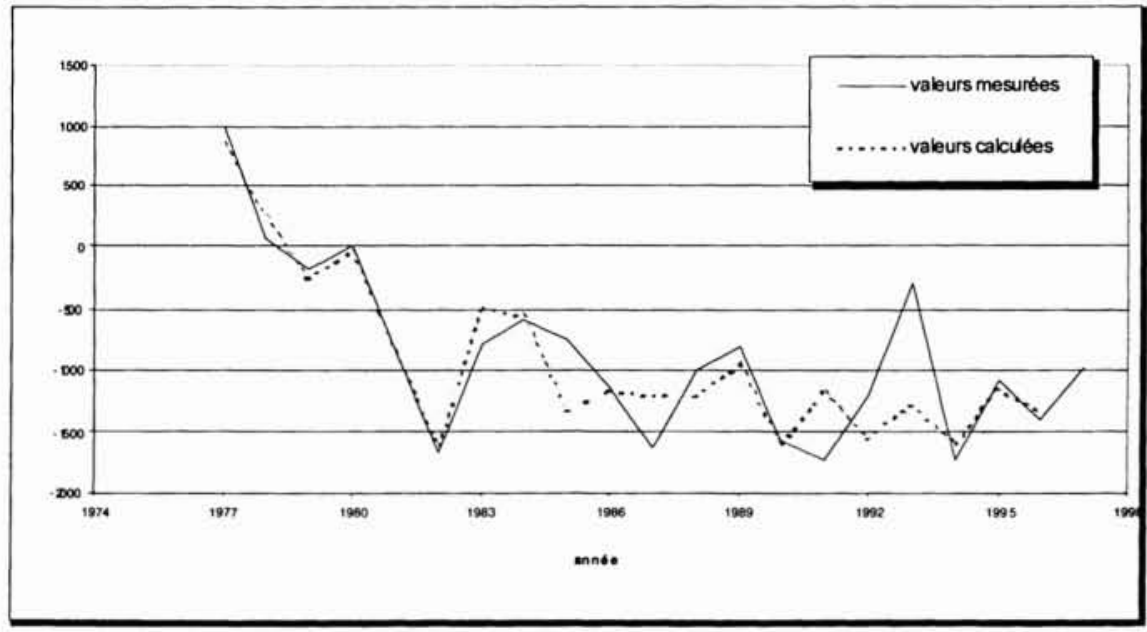

où:

$K_{d}=$ radiation diffuse moyenne mensuelle

$s \quad=$ inclinaison de la surface par rapport à l'horizon

$\rho=$ réflectance du terrain (variable entre 0,2 et 0,7 selon l'extension de la couverture neigeuse)

$R_{b}=$ rapport entre la radiation mensuelle incidente sur une surface inclinée par rapport à celle sur une surface horizontale.

Cette expression contient explicitement les termes relatifs au rapport entre la radiation directe et celle diffuse, alors que les relations trigonométriques complexes sont contenues dans le terme $R_{b}$, pour lequel nous renvoyons à ([5]. Les valeurs mensuelles de la radiation globale incidente sur la surface ayant une pente et une orientation correspondantes à celles moyennes du glacier examiné, ont permis de calculer les valeurs d'énergie accumulée du glacier:

$$
K_{t}^{\prime}=K_{t}(1-a)
$$

où $a$ désigne l'albédo (auquel on a donné la valeur moyenne de 0,8 ). La valeur de la chaleur latente a permis de convertir ces valeurs en $\mathrm{kg} / \mathrm{m}^{2}$ équivalents de glace fondue ; on a pu alors déterminer les valeurs annuelles grâce à la somme des valeurs mensuelles pendant la période juin-octobre.

En soustrayant à ces valeurs la quantité d'eau équivalente à l'épaisseur de la couverture neigeuse de la fin du mois de mai (à laquelle nous avons assigné une densité moyenne de
$600 \mathrm{~kg} / \mathrm{m}^{3}$ ), on obtient un prédicteur (appelé "RAD_ACC") qui représente physiquement et approximativement, l'épaisseur de la neige ou de la glace enlevée par l'action de l'énergie radiante accumulée par la surface du glacier, pendant toute la période d'ablation (c'est-à-dire le terme $\Sigma_{T}\left(R-a_{c}\right)$ de l'équation (3)).

Les expériences précédentes menées sur la série du bilan de masse net de la Vedretta du Caresèr ([6] ont permis de conclure que le prédicteur dérivé du terme d'advection s'obtient en calculant les valeurs de degré jours $>0^{\circ} \mathrm{C}$ de la période d'ablation (mai-octobre) de chaque année, à partir des températures moyennes journalières égales à la moyenne pondérées des températures du jour ( $1 / 3$ température minimale $+2 / 3$ température maximale), calculées à l'altitude de 3000 mètres avec un gradient de température vertical déterminé expérimentalement.

La variable ainsi calculée est symbolisée par "DD_3000" (et on a remarqué que pour les glaciers ici étudiés elle peut être approchée par l'expression $\Sigma_{T}\left(T_{a}-T_{s}\right)$ de l'équation (3)).

\section{III — LE BILAN DE MASSE DES GLACIERS DU GROUPE ORTLES-CEVEDALE}

Le calibrage d'un modèle dont le bilan de masse spécifique (indiqué "BIL_SP") représente la variable indépendante, avec 17 couples des valeurs sur 21 disponibles (c'est-à-dire en excluant quatre couples des valeurs "outliers"), est donné par la formule suivante : 
4. Modèle à 3 variables du Glacier de la Sforzellina Graphique de dispersion des valeurs simulées/mesurées.

5. Modèle à 3 variables du Glacier de Fontana Bianca Graphique de dispersion des valeurs simulées/mesurées.
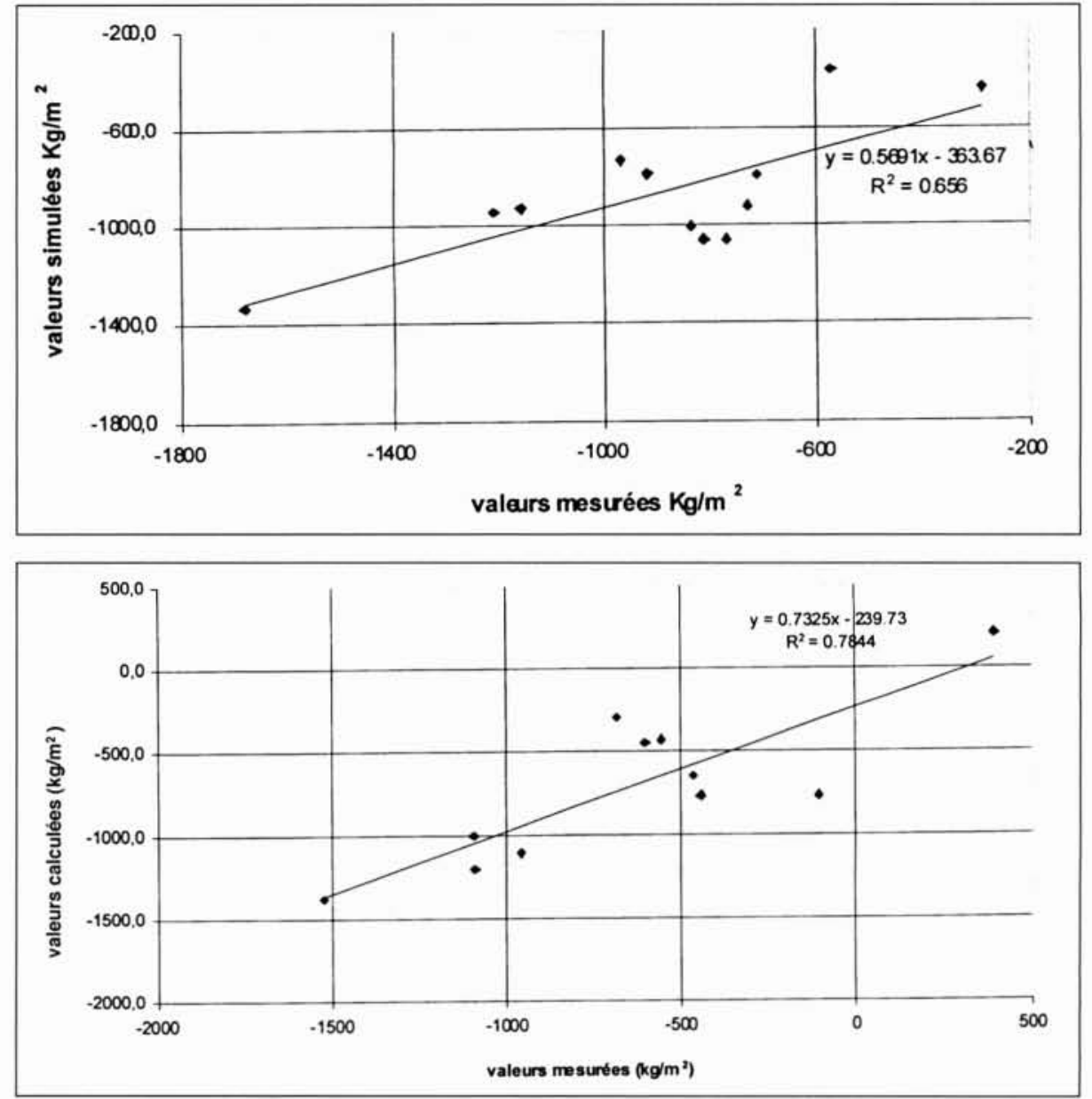

BIL_SP $=-1,1803$ RAD_ACC $+137,23 \quad$ avec $\mathrm{R}^{2}=0,919$

En considérant la forte corrélation entre les séries de bilan de masse des glaciers, objet de cette étude, avec la série du glacier autrichien Hintereisferner on a décidé d'introduire une deuxième variable indépendante (dénommée pour abréger "HINTEREIS") dans le modèle exposé ci-dessus, dans le but de réduire l'indétermination liée aux événements qui échappent au modèle. En ajoutant cette nouvelle variable, le calibrage des paramètres sur la série réduite des données $(17 / 21)$ a donné le résultat suivant :

$$
\begin{aligned}
& \text { BIL_SP }=-0,8579 \text { RAD_ACC }+0,3795 \text { HINTEREIS }+ \\
& 60,706 \\
& \text { avec } R^{2}=0,9172 \text {. }
\end{aligned}
$$

La variable la plus représentative est en tout cas "RAD_ACC", qui représente à peu près $70 \%$ de la variance, alors que la variable "HINTEREIS" représente les $30 \%$ restant. On a rapporté respectivement sur les figures 2 et 3 les graphiques de dispersion des valeurs simulées/mesurées, relatives au calibrage sur la série réduite des données (17/21) pour la comparaison des séries temporelles.

Les deux glaciers restants du Groupe Ortles-Cevedale présentent une structure de la série temporelle du bilan de masse moins continuelle et souvent sans les valeurs d'accumulation. Les résultats des bilans de ces glaciers ont presque tous montré que l'altitude de l'ELA fut plus élevée que celle maxi- male, ce qui réduit la signification statistique de cette relation. Même pour ces glaciers, les variables "DD_3000" et "RAD_ACC" ont été calculées. Pour ce qui concerne le Glacier de la Sforzellina, dont la série du bilan commence en 1987 [7], la variable "DD_3000" a été obtenue en calculant les valeurs positives de degré*jours $>0^{\circ} \mathrm{C}$ à 3000 mètres avec les températures quotidiennes relevées à la station de $S$. Caterina Valfurva, station qui se trouve à une altitude de 1740 mètres ; la variable "RAD_ACC" a été calculée, en l'absence des données analogues relatives aux stations de la Valtellina, en utilisant les observations sur l'état du ciel de la station Diga Caresèr, alors que pour la hauteur du manteau de neige on a fait référence à la station de S. Caterina Valfurva 2000, qui se trouve à une altitude décidément trop basse pour être représentative d'une manière adéquate des conditions microclimatiques qui ont influencé le bilan du glacier examiné. Le modèle qui en résulte, à 3 paramètres, a la forme suivante :

$$
\begin{aligned}
\text { BIL_SP }= & 0,440 \text { RAD_ACC }-1,997 \text { DDAY_3000 + } \\
& 0,7495 \text { HINTEREIS }+365,00 \quad \text { où } R^{2}=0,656
\end{aligned}
$$

La faible qualité du résultat obtenu, en considérant l'utilisation de 3 paramètres, est probablement due, premièrement à l'utilisation des données climatiques de stations éloignées du glacier et donc peu représentatives des conditions, et deuxièmement par le peu de données numériques fournis par la série des mesures de bilan (13 ans de mesures contre les 21 données au Caresèr). 
Les prédicteurs, qu'il faut utiliser dans le modèle pour le Glacier de Fontana Bianca, dont la série des mesures de bilan commence en 1984 [8], mais qui présente une interruption de deux ans, ont été calculés en utilisant les données météorologiques qui proviennent exclusivement de la station de Diga Lago Verde.

Dans ce cas le modèle obtenu, toujours à 3 paramètres. présente la forme suivante :

$$
\text { BIL_SP }=0,0626 \text { RAD_ACC }-0,327 \text { DDAY_3000 + }
$$$$
1,307 \text { HINTEREIS + 123,9 où } \mathrm{R}^{2}=0,787 \text {. }
$$

Les résultats obtenus sont assez positifs, même avec des considérations analogues à celles exposées ci-dessus pour le Glacier de la Sforzellina. Pour une estimation comparative des résultats obtenus à partir de l'application des deux modèles aux glaciers de la Sforzellina et de Fontana Bianca, sont montrés les diagrammes de dispersion des valeurs calculées/mesurées, fiables à $95 \%$, respectivement sur la figure 4 et 5 .

\section{IV $\square$ CONCLUSION}

L'application des modèles décrits et définis semi-empiriques à base physique, aux séries temporelles des mesures de bilan de masse des glaciers du Groupe Ortles-Cevedale, met en évidence premièrement que les résultats sont d'autant plus significatifs que les données au début sont fiables, surtout si on considère des petits glaciers dans lesquels le signal causé par les variables indépendantes (facteurs forçants) à une échelle régionale s'ajoute aux influences locales. Une description correcte des termes radiatifs, possible grâce aussi aux données de base simples, mais correctes, est fondamentale pour la réussite de ces modèles. De ces résultats on déduit aussi l'exigence d'une récupération critique, par l'ana- lyse sur la congruence des données, de quelques séries temporelles des unités de grandeur météorologiques, incluant même les observations sur l'état du ciel, utiles pour les modèles à utiliser dans la reconstitution des valeurs de bilan de masse pour les glaciers sur lesquels est disponible une série adéquate de mesures.

\section{BIBLIOGRAPHIE}

II] Kunn M.. (1981): Climate and glaciers. Proc. of the IAHS Canberra Symp. on Sea Level, Ice and Climatic Change, Dec. 1979. IAHS Publ. $n^{\circ}$ 131, pp. 3-20.

[2] LIU B.Y.H., JORDAN R.C. (1962): Daily insolation on surfaces tilted toward the equator. Trans. ASHRAE pp. 325-329.

13] PAGE K.J. (1961): The estimation of monthly mean values of daily shortwave radiation on vertical and inclined surfaces from sunshine record for latitude $40^{\circ} \mathrm{N}-40^{\circ} \mathrm{S}$. Proc. UN Conf, on New Sources of Energy. Paper No. 35/5/98.

14] Stull. R.B. (1997): An introduction to Boundary Layer Meteorology. Kluwer Ac. Publishers, pp. 253:260. Revised ed. 1997.

[5] K.EIN S.S. (1977): Monthly average insolation on tilted surfaces. Solar Energy. Vol. 19 (325-329).

[6] Rossı G.C.. Zanon G., Smiragian C., Rossi S. (2000): Evoluzione tren tennale del bilancio di massa della Vedretta del Caresèr in relazione alle condizioni climatiche. Atti Seminario "Bilanci di massa dei ghiacciai. Metodi e prospettive". Rifugio Larcher (TN), 11 - 12 settembre 1997.

17] Catasta G. . Smiragla C. (1993) - The mass balance of a cirque glacier in the Italian Alps (Ghiacciaio della Sforzellina). Journ. Glac., 39. $87-90$.

[8] Kaser G.. Munarı M. (1996): Glacier Mass Balance Bulletin, Bull. No 4 (1994-1995). W.Haeberli, M. Hoelzle, S. Suter Eds.

[9] Rossi G.C.. ZANon G. (1994): Impact of the recent glacier retreat on the hydrology of an alpine basin. Proc. IAHR Ice Symp. Trondheim 1994.

[10] Zanon G.. (1992): Venticinque anni di bilancio di massa del Ghiacciaio del Caresèr, 1966-67/1990-91. Geogr. Fis. Dinam. Quat., 15.215-219. 